\title{
Flexible Capacity Sharing in Multi-tenant Wireless Networks through Fuzzy Neural Controllers
}

\author{
J. Pérez-Romero, O. Sallent, R. Ferrús, R. Agustí \\ Dept. of Signal Theory and Communications \\ Universitat Politècnica de Catalunya (UPC) \\ Barcelona, Spain \\ \{jorperez, sallent, ferrus, ramon\}@tsc.upc.edu
}

\begin{abstract}
The introduction of multi-tenancy in the Radio Access Network (RAN) is seen as a relevant capability of future 5G systems to support the challenging capacity requirements in a cost-effective way. Multi-tenancy brings in new challenges in the way how the RAN has to be managed and operated in order to provide the agreed service levels to each tenant and at the same time achieve an efficient utilization of the radio resources. This paper proposes a new solution for flexible capacity sharing among tenants based on a hybrid centralized/distributed SelfOrganizing Network (SON) function to automatically adjust the Admission Control (AC) settings of the different cells. The proposed solution makes use of fuzzy neural controllers that provide intrinsic benefits in terms of dealing with the uncertainties of complex cellular scenarios and introducing the formulation of preferences and policies in the decision process. Together with the detailed description of the different components of the proposed solution, the paper presents an initial evaluation that provides sufficient insight into the potentials of the fuzzy neural hybrid SON to stimulate further and subsequent analysis.
\end{abstract}

Keywords-Multi-tenancy; RAN sharing; Self-Organizing Networks (SON); Fuzzy Neural control; Admission Control

\section{INTRODUCTION}

Multi-tenant Radio Access Networks (RAN), in which the wireless network infrastructure may be deployed and operated by an specialized infrastructure provider and its access capacity shared among several communication providers, i.e. "tenants", are envisaged to become a key element of future Fifth Generation $(5 \mathrm{G})$ systems to support the challenging capacity requirements, since the sharing process will reduce both capital and operational costs [1]. In this respect, the main enablers for realizing future multi-tenant networks are presented in [2] as an evolution starting from the standardization activities on network sharing carried out by the 3rd Generation Partnership Project (3GPP).

An important challenge in a multi-tenant RAN is the optimization of the network configuration to ensure that, on the one hand, the available capacity is properly distributed among tenants so that each one gets the expected service and, on the other hand, that deployed radio resources are used efficiently.

In this framework, the objective of this paper is to propose a new strategy for flexible capacity sharing in multi-tenant RANs to efficiently regulate the amount of traffic of each tenant that can be admitted in the network. In contrast to other works, such as [3]-[6], which have focused on the scheduling process to split the radio resources of a RAN among tenants, the proposed solution enforces the network capacity sharing targets by means of a Self-Organizing Network (SON) function that acts on the Admission Control (AC) process for Guaranteed Bit Rate (GBR) traffic. The proposed SON approach makes use of fuzzy neural (FN) decision making in order to deal with the vagueness and uncertainty that are characteristics of complex cellular scenarios due to the effects of random channel variations, user mobility, traffic generation dynamics, etc. Furthermore, it allows introducing in the decision making process the non-specificity inherent to the human formulation of preferences and policies of operation.

Different applications of fuzzy logic and neural networks exist in the context of Radio Resource Management (RRM) for wireless networks, covering aspects such as handover [7][8][9], admission control [10][11], power control [12] or Radio Access Technology selection [13]. However, to the authors' best knowledge, there are no prior works that have applied FN in the area of SON for multi-tenant networks.

Regarding the use of SON techniques for self-optimization of AC, literature such as [14]-[18] has considered a traditional RAN owned and operated by a single operator. However, the consideration of multi-tenancy introduces a fundamental difference in the way how these techniques have to be designed, because they need to incorporate additional dimensions to capture the specificities of each tenant (e.g. the different service expected in accordance with the Service Level Agreements [SLAs]) and the possible complementarities between tenants (e.g. the spatial and time distribution of each tenant may not be the same across the whole RAN).

Sustained on the novelties highlighted above, the rest of the paper is organized as follows. Section II presents the general framework for the proposed hybrid SON solution for capacity sharing. Its two components, namely the centralized and the decentralized SON are described, respectively, in Sections III and IV. Section V presents a performance evaluation and Section VI concludes the paper.

\section{HYBRID SON FOR CAPACITY SHARING}

Let us consider a RAN with multiple cells deployed and operated by an infrastructure provider, which is shared among $S$ tenants numbered as $s=1, \ldots, S$. Each tenant offers services to their own customers. From a service perspective, the RAN provides Radio Access Bearers (RABs), which are the data delivery services offered across the RAN for information exchange between the User Equipment (UE) and the mobile Core Network $(\mathrm{CN})$. A SLA between each tenant and the infrastructure provider specifies the aggregated GBR to be 
provided for the set of the tenant's RABs activated across all of the deployed cells.

Based on the SLAs from all tenants, it is assumed that the infrastructure provider has deployed $N$ cells numbered as $n=1, \ldots, N$ in a certain area, whose capacity should fit the tenants' needs. Nevertheless, during the network operation stage, the actual offered traffic of the tenant $s$ in cell $n$ will vary along the time and exhibit discrepancies with respect to the planned traffic level. Therefore, it has to be ensured that the deployed cell capacity is properly shared among the tenants according to their SLA and at the same time that this capacity is efficiently utilized.

To this end, the functional architecture of the proposed solution is shown in Fig. 1. On the one hand, an AC function, executed at each cell in line with current RAN architectures (e.g. in Long Term Evolution [LTE]), is considered to control the served traffic of each tenant by deciding on the acceptance or rejection of new GBR RAB establishments in that cell. On the other hand, a SON function is introduced to dynamically and coordinately modify the behavior of the AC across all the RAN cells in order to fulfil the target network aggregate behavior for all the tenants.

From an architectural perspective, as discussed in [19][20], SON functionalities can be implemented as centralized SON (cSON) solutions being part of the Network Management System (NMS) and/or the Element Management System (EMS) of the deployed network infrastructure, as distributed SON (dSON) solutions where the SON algorithms run at the radio network equipment (e.g. the cells) or as hybrid SON solutions that combine both $\mathrm{cSON}$ and dSON components. In the hybrid SON, the cSON component provides guidelines and parameter ranges to the $\mathrm{dSON}$ functions, based on information retrieved from them in terms of e.g. performance measurements. Then the local dSON functions adjust local parameter settings within the provided ranges.

In general, SON algorithms requiring fast response, UE specific information and rich input data sets are more easily implemented locally by dSON, while SON algorithms requiring wide area visibility and parameter settings encompassing multiple cells, without the need for fast response times, are more easily implemented by cSON. In turn, a hybrid SON architecture combines the best of the two approaches [20].

Given that the design of an efficient capacity sharing mechanism requires both the wide area/long term picture (i.e., assessing how the traffic load is being distributed among tenants over the whole scenario in order to ensure that capacity is fairly split in the long term) as well as the local area/short term picture (i.e., assessing how the traffic is being offered on a cell level basis by each tenant in order to ensure that traffic fluctuations below the average of one tenant can be exploited by traffic fluctuations above the average from another tenant), the hybrid SON architecture is considered in this paper. Specifically, the cSON in the proposed approach establishes a limit in the maximum fraction of load of each tenant $s$ that should be blocked in each cell $n$ based on the overall offered load of all the tenants in the different cells. This is specified as a maximum blocking probability denoted as $P_{B, \max }(s, n)$. Based on this information the $\mathrm{dSON}$ at cell $n$ will finally decide the specific blocking probability $P_{B}(s, n)$ in the range $\left[0, P_{B, \max }(s, n)\right]$ taking into consideration local conditions of each tenant (e.g. bit rate of the users of the tenant in the cell, resource consumption, etc.). Both cSON and dSON are designed as FN controllers.

Finally, the AC function will make the acceptance or rejection of the new $\mathrm{RAB}$ establishments requests in order to fulfil the blocking probability $P_{B}(s, n)$ decided by the dSON. In the case that all the RABs of tenant $s$ have the same priority, the AC algorithm at the $n$-th cell can consist simply in statistically rejecting connections of the $s$-th tenant with probability $P_{B}(s, n)$. In turn, when the RABs belong to services with different priority, a similar process can be applied by decomposing first the probability $P_{B}(s, n)$ in a set of different probabilities for each priority class while keeping $P_{B}(s, n)$ on overall terms.

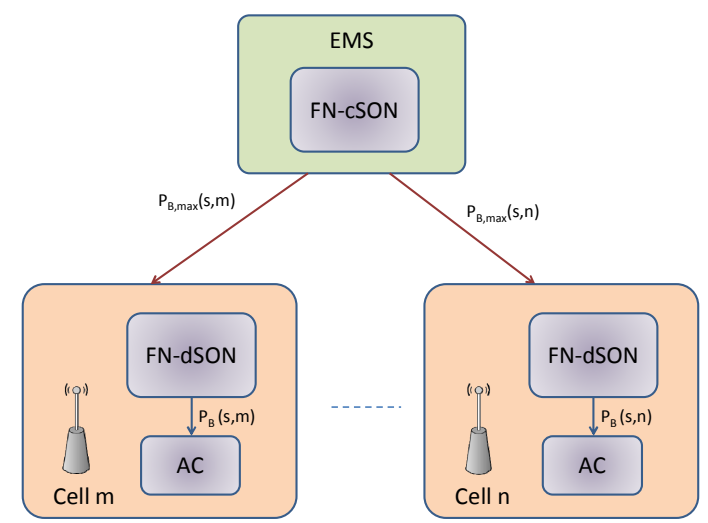

Fig. 1. Functional architecture of the Fuzzy Neural Hybrid SON approach

\section{FUZZY NEURAL CSON}

The Fuzzy Neural cSON (FN-cSON) consists of a FN controller per tenant and cell responsible of deciding the values of $P_{B, \max }(s, n)$. Each controller consists of a fuzzy-based decision making process represented by means of a feedforward neural network of 5 layers depicted in Fig. 2. The different layers of the feed-forward process carry out the fuzzification, inference engine and defuzzification steps of fuzzy logic starting from a set of input linguistic variables.

The linguistic variables considered by the FN-cSON correspond to different measurements of the offered load of the different tenants in the different cells. Specifically, the following three linguistic variables are considered.

- Offered load of tenant $s$ in cell $n$ : This measurement is given by the offered traffic of the tenant in the cell $T(s, n)$ relative to the planned average traffic $T_{p}(s, n)$ that was considered by the infrastructure provider when deploying the cell. Then, the measurement is defined as $L_{c}(s, n)=T(s, n) / T_{p}(s, n)$.

- Offered load of all the other tenants $s$ different from $s$ in cell $n$ : This measurement captures the offered traffic of the other tenants in relation to their planned traffic. It is denoted as $L_{c}(-s, n)$ and is given by:

$$
L_{c}(-s, n)=\frac{\sum_{s^{\prime} \neq s} T\left(s^{\prime}, n\right)}{\sum_{s^{\prime} \neq s} T_{p}\left(s^{\prime}, n\right)}
$$


- Offered load of tenant $s$ in all the other cells $n$ ' different from $n$. This is denoted as $L_{c}(s,-n)$ and is defined as the average of $L_{c}(s, n$ ') for all the other cells:

$$
L_{c}(s,-n)=\frac{1}{N-1} \sum_{n^{\prime} \neq n} L_{c}\left(s, n^{\prime}\right)
$$

The three variables $L_{c}(s, n), L_{c}(-s, n)$ and $L_{c}(s,-n)$ correspond to average values within the time window of operation of the cSON. Each linguistic variable is expressed by means of a term set composed of 3 fuzzy sets. They are denoted as: L ("Low"), i.e. the load is below the planned level; P ("Planned"), i.e. the load is approximately equal to the planned level; and $\mathrm{H}$ ("High"), i.e. the load is above the planned level.

As illustrated in Fig. 2, each neuron of Layer 1 represents one of the input linguistic variables, and is connected to three neurons of Layer 2 associated to the fuzzy sets of this variable.

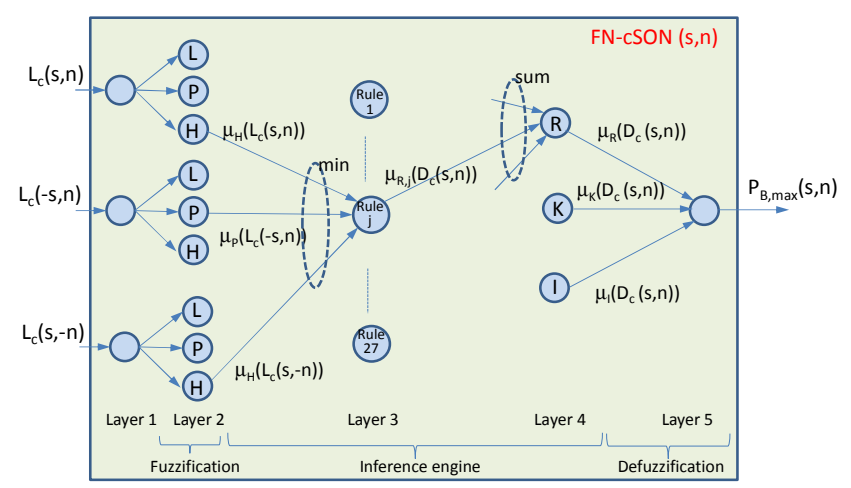

Fig. 2. Fuzzy Neural cSON controller for tenant $s$ and cell $n$

\section{A. Fuzzification}

The fuzzification process is performed by the neurons of Layer 2 in Fig. 2 and computes the degree of membership of each input linguistic variable to the $\mathrm{L}, \mathrm{P}$ and $\mathrm{H}$ fuzzy sets. The degree of membership is denoted as $\mu_{X}(y)$ where $y$ represents the input linguistic variable (i.e. $L_{c}(s, n), L_{c}(-s, n)$ or $\left.L_{c}(s,-n)\right)$ and $X$ the fuzzy set, i.e. $X \in\{\mathrm{L}, \mathrm{P}, \mathrm{H}\}$. Bell-shaped Gaussian membership functions are considered here. They are defined as follows for the fuzzy sets $\mathrm{L}, \mathrm{P}$ and $\mathrm{H}$, respectively:

$$
\begin{gathered}
\mu_{L}(y)=\left\{\begin{array}{lll}
1 & \text { if } & y<m_{L} \\
e^{-\left(\frac{y-m_{L}}{\sigma_{L}}\right)^{2}} & \text { if } & y \geq m_{L}
\end{array}\right. \\
\mu_{P}(y)=e^{-\left(\frac{y-m_{P}}{\sigma_{P}}\right)^{2}} \\
\mu_{H}(y)=\left\{\begin{array}{lll}
e^{-\left(\frac{y-m_{H}}{\sigma_{H}}\right)^{2}} & \text { if } & y \leq m_{H} \\
1 & \text { if } & y>m_{H}
\end{array}\right.
\end{gathered}
$$

The parameters $m_{X}, \sigma_{X}$ with $X \in\{\mathrm{L}, \mathrm{P}, \mathrm{H}\}$ are the center and width of the Gaussian membership function of fuzzy set $X$. These parameters can be set differently depending on the considered linguistic variable being fuzzified, and can be set differently in the controllers of different tenants and cells.

\section{B. Inference Engine}

The inference engine specifies the behavior of the FN controller by defining a set of inference rules or decision policies. Each rule is characterized by a linguistic IF-THEN proposition that, for a certain combination of the fuzzy sets of the input variables, infers a consequence. The consequence considered here is denoted as $D_{c}(s, n)$ and reflects the amount of traffic that should be admitted for tenant $s$ in cell $n$. It is expressed by means of the following 3 fuzzy sets: $R$ ("Reduce"), meaning that the number of admissions has to be decreased; K ("Keep"), meaning that the number of admissions has to be maintained; and I ("Increase"), meaning that the number of admissions has to be increased.

The number of inference rules is given by the number of possible combinations of the fuzzy sets associated to the input linguistic variables. Then, since there are 3 input variables and each one is defined by 3 fuzzy sets $\{\mathrm{L}, \mathrm{P}, \mathrm{H}\}$, the number of rules is $3^{3}=27$. In the neural network structure of Fig. 2 each neuron of Layer 3 represents an inference rule. It is connected to 3 neurons of Layer 2 (i.e. one per fuzzy set of an input linguistic variable) and receives from them the values of the membership functions of the input linguistic variables. In turn, the neurons of Layer 4 represent the 3 fuzzy sets of the inferred consequence, i.e. $\{\mathrm{R}, \mathrm{K}, \mathrm{I}\}$. Therefore, each neuron of Layer 3 (i.e. each inference rule) is connected to one neuron of Layer 4 that corresponds to the inferred consequence of the rule.

For example, the rule $j$ illustrated in Fig. 2 corresponds to the proposition: IF $\left[\left(L_{c}(s, n)\right.\right.$ is "High") AND $\left(L_{c}(-s, n)\right.$ is "Planned") AND $\left(L_{c}(s,-n)\right.$ is "High")] THEN $\left[D_{c}(s, n)\right.$ $=$ "Reduce" admission for tenant $s$ in cell $n$ ].

The membership value of the decision $D_{c}(s, n)$ for each of the fuzzy sets $\{\mathrm{R}, \mathrm{K}, \mathrm{I}\}$ in a given rule $j$ is defined as the minimum among the membership values of the input fuzzy sets considered in this rule. This operation is performed by the Layer 3 neurons and is expressed as:

$$
\mu_{z, j}\left(D_{c}(s, n)\right)=\min \left(\mu_{X_{1, j}}\left(L_{c}(s, n)\right), \mu_{X_{2, j}}\left(L_{c}(-s, n)\right), \mu_{X_{3, j}}\left(L_{c}(s,-n)\right)\right)(6)
$$

where $Z \in\{\mathrm{R}, \mathrm{K}, \mathrm{I}\}$ is the fuzzy set of the inferred consequence in rule $j$ (i.e. $\mathrm{Z}=\mathrm{R}$ in the example of Fig. 2) while $X_{1, j}, X_{2, j}, X_{3, j}$ $\in\{\mathrm{R}, \mathrm{K}, \mathrm{I}\}$ are the fuzzy sets of the input linguistic variables $L_{c}(s, n), L_{c}(-s, n), L_{c}(s,-n)$ considered by rule $j$, respectively, (i.e. $X_{1, j}=\mathrm{H}, X_{2, j}=\mathrm{P}, X_{3, j}=\mathrm{H}$ in the example of Fig. 2).

The value of $\mu_{Z, j}\left(D_{c}(s, n)\right)$ is passed from the Layer 3 neuron corresponding to rule $j$ to the Layer 4 neuron corresponding to the inferred consequence $Z$. Then, the Layer 4 neurons combine the values $\mu_{Z, j}\left(D_{c}(s, n)\right)$ from multiple inference rules in order to compute the total membership value $\mu_{Z}\left(D_{c}(s, n)\right)$ of the fuzzy set $Z \in\{R, K, I\}$. Specifically, this membership value is computed using a fuzzy OR operation as [21]:

$$
\mu_{Z}\left(D_{c}(s, n)\right)=\min \left(1, \sum_{j \in C_{Z}} \mu_{Z, j}\left(D_{c}(s, n)\right)\right)
$$

where $C_{Z}$ is the set of rules whose inferred consequence is $Z \in\{\mathrm{R}, \mathrm{K}, \mathrm{I}\}$.

\section{Defuzzification}

The defuzzification process consists in converting the outputs of the inference engine into a crisp value that 
represents the output of the decision, i.e. the maximum blocking probability $P_{B, \max }(s, n)$. The defuzzification method considered here is the center-of-area method [22], which can be expressed as follows:

$$
P_{B, \max }(s, n)=\frac{\sum_{Z \in\{R, K, I\}} m_{Z} \cdot \sigma_{Z} \cdot \mu_{Z}\left(D_{c}(s, n)\right)}{\sum_{Z \in\{R, K, I\}} \sigma_{Z} \cdot \mu_{Z}\left(D_{c}(s, n)\right)}
$$

The parameters $m_{Z}, \sigma_{Z}$ are the centers and widths of membership functions associated to the fuzzy set $Z \in\{R, K, I\}$ and they are parameters to be set. It can be easily seen that, assuming that $m_{R} \geq m_{K} \geq m_{I}$ the expression (8) is constrained to the range $\left[m_{I}, m_{R}\right]$. Therefore, in order to have a realizable blocking probability, it should be fulfilled that $m_{R} \leq 1$ and $m_{l} \geq 0$.

\section{FUZZY NEURAL DSON}

The Fuzzy Neural - dSON (FN-dSON) is executed at each cell and makes use of the outcome of the FN-cSON to decide the blocking probability $P_{B}(s, n)$ to be applied to each tenant. While the FN-cSON decisions consider the situation of the different tenants across the whole scenario, the FN-dSON decisions are based on local information at each cell. Therefore the FN-dSON can adapt the decisions to the specificities of each cell in terms of load, propagation, interference, etc. Besides, the FN-dSON will operate in general with a shorter time scale than the FN-cSON, so that it can adapt to traffic fluctuations.

The FN-dSON at the $n$-th cell consists of one fuzzy neural controller like the one presented in Fig. 3 for each tenant. The general structure of the controller is similar to that of the FNcSON.

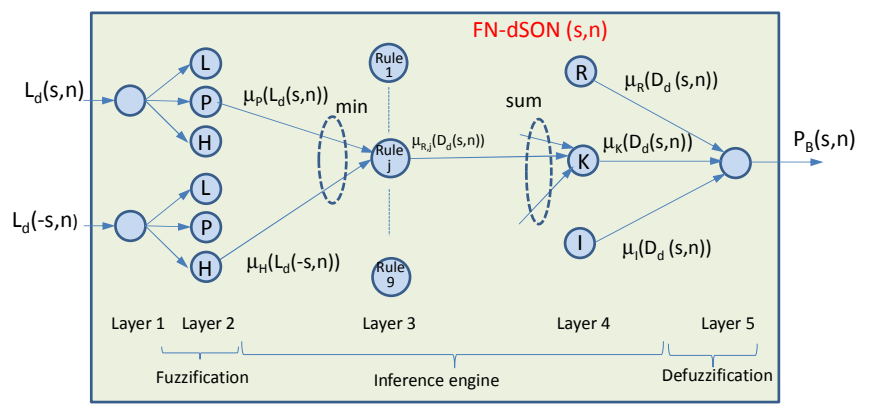

Fig. 3. Fuzzy Neural dSON controller for tenant $s$ in cell $n$

The input linguistic variables of the FN-dSON are local to each cell. The considered values include only the offered load of a tenant and the offered load of the other tenants are considered. These variables are denoted as $L_{d}(s, n)$ and $L_{d}(-s, n)$ and are defined in a similar way as the $L_{c}(s, n)$ and $L_{c}(-s, n)$ variables of the FN-cSON, respectively, although they are average values over the time scale of operation of the $\mathrm{FN}$ dSON. The fuzzification of these two variables makes use of similar functions like those of (3)-(5) but with specific parameters $m_{X}, \sigma_{X}$ set locally.

The inference engine in this case contains just a set of 9 inference rules (i.e. 2 linguistic variables, each one with 3 fuzzy sets, thus leading to $3^{2}=9$ rules). The inferred consequence is denoted as $D_{d}(s, n)$ and its membership value is computed by the Layer 4 neurons making use of similar functions to $(6)(7)$. It is worth mentioning that, even if the two linguistic variables are the same as in the FN-cSON, the rules of the FN-dSON are in general different, because it operates at a different time scale and it has to take local decisions for the $n$-th cell.

The defuzzification process is used to determine the value of $P_{B}(s, n)$ taking into consideration the limit $P_{B, \max }(s, n)$ set by the FN-cSON. This is achieved by particularizing (8) with $m_{R}=$ $P_{B, \max }(s, n)$ :

$$
P_{B}(s, n)=\frac{P_{B, \max }(s, n) \cdot \sigma_{R} \cdot \mu_{R}\left(D_{d}(s, n)\right)+\sum_{z \in\{K, I\}} m_{z} \cdot \sigma_{z} \cdot \mu_{z}\left(D_{d}(s, n)\right)}{\sum_{Z \in\{R, K, I\}} \sigma_{z} \cdot \mu_{z}\left(D_{d}(s, n)\right)}
$$

It can be easily shown that, by setting $m_{R}=P_{B, \max }(s, n)$ $\geq m_{K} \geq m_{l}=0$ the above expression is always constrained to the range $\left[0, P_{B, \max }(s, n)\right]$.

\section{Performance Evaluation}

This section provides a first insight on the performance that can be obtained with the proposed framework. The objective of the analysis is to validate the capabilities of the FN-cSON and FN-dSON to drive the decision making towards satisfactory capacity sharing among tenants and efficient resource usage in front of different traffic mixes reflecting different traffic spatial distributions.

The evaluation assumes the outdoor Urban Micro scenario of [23] with cell inter-site distance $200 \mathrm{~m}$ and hexagonal layout. Each cell has one LTE carrier of $10 \mathrm{MHz}$, organized in a total of 50 Physical Resource Blocks (PRBs) of $180 \mathrm{kHz}$. The operation frequency is $2.6 \mathrm{GHz}$. Path loss and shadowing models are from [23]. The downlink direction is considered with transmitted power per PRB $24 \mathrm{dBm}$ and UE noise figure 9 $\mathrm{dB}$. The spectral efficiency model to map Signal to Interference and Noise Ratio and bit rate is taken from section A.1 of [24] with a maximum spectral efficiency of $4.4 \mathrm{~b} / \mathrm{s} / \mathrm{Hz}$. With these parameters, the peak traffic that can be supported by the cell is $39.6 \mathrm{Mb} / \mathrm{s}$ (i.e. $50 \mathrm{PRBs}$ of $180 \mathrm{kHz}$ with the maximum spectral efficiency).

The offered traffic of each tenant is modelled using a Poisson arrival model and exponential session duration with average 30s and GBR $1024 \mathrm{~kb} / \mathrm{s}$. For an easier interpretation of the results, a scenario with $N=2$ cells -each operating in a different carrier- and $S=2$ tenants, denoted as T1 and T2, is considered. Based on the SLAs with each tenant and the considered deployment, the infrastructure provider sets the planned average traffic in all the cells equal to $T_{p}(1, n)=12.5$ $\mathrm{Mb} / \mathrm{s}$ and $T_{p}(2, n)=18.5 \mathrm{Mb} / \mathrm{s}$ for tenants $\mathrm{T} 1$ and $\mathrm{T} 2$, respectively.

The FN-cSON and FN-dSON controllers are configured with the inference rules shown in Table I and Table II. The parameters of the membership functions of the Layer 2 neurons for both controllers are set as $m_{L}=0.5, \sigma_{L}=0.5, m_{P}=1, \sigma_{P}=0.5$, $m_{H}=1.5$, and $\sigma_{H}=0.5$. The Layer 5 neurons of the FN-cSON controllers are configured with $m_{I}=0.001, \sigma_{I}=0.02, m_{K}=0.02$, $\sigma_{K}=0.02$ and $\sigma_{R}=0.1$. In turn, the value of $m_{R}$ for the FN-cSON controllers is adjusted depending on the offered and planned traffic of each tenant $s$ in each cell $n$ to establish a reference blocking probability level that would reject the traffic in excess 
of the planned level ${ }^{1}$. This leads to $m_{R}(s, n)=\max (1-$ $\left.T_{p}(s, n) / T(s, n), 0.04\right)$. The parameters of the Layer 5 neurons of the FN-dSON controllers are set in (9) as $m_{l}=0, \sigma_{I}=0.02$, $m_{K}=0.02, \sigma_{K}=0.02$ and $\sigma_{R}=0.1$.

TABLE I. INFERENCE RULES OF THE FN-CSON

\begin{tabular}{|c|c|c|c|c|c|c|c|}
\hline$L_{c}(s, n)$ & $L_{c}(-s, n)$ & $L_{c}(s,-n)$ & $D_{c}(s, n)$ & $L_{c}(s, n)$ & $L_{c}(-s, n)$ & $L_{c}(s,-n)$ & $D_{c}(s, n)$ \\
\hline$\overline{\mathrm{L}}$ & $\overline{\mathrm{L}}$ & $\overline{\mathrm{L}}$ & I I & $\overline{\mathrm{P}}$ & $\overline{\mathrm{P}}$ & $\overline{\mathrm{H}}$ & $\overline{\mathrm{K}}$ \\
\hline $\mathrm{L}$ & $\mathrm{L}$ & $\mathrm{P}$ & $\mathrm{I}$ & $\mathrm{P}$ & $\mathrm{H}$ & $\mathrm{L}$ & $\mathrm{K}$ \\
\hline $\mathrm{L}$ & $\mathrm{L}$ & $\mathrm{H}$ & $\mathrm{I}$ & $\mathrm{P}$ & $\mathrm{H}$ & $\mathrm{P}$ & $\mathrm{K}$ \\
\hline $\mathrm{L}$ & $\mathrm{P}$ & $\mathrm{L}$ & $\mathrm{I}$ & $\mathrm{P}$ & $\mathrm{H}$ & $\mathrm{H}$ & $\mathrm{K}$ \\
\hline $\mathrm{L}$ & $\mathrm{P}$ & $\mathrm{P}$ & I & $\mathrm{H}$ & $\mathrm{L}$ & $\mathrm{L}$ & $\mathrm{K}$ \\
\hline $\mathrm{L}$ & $\mathrm{P}$ & $\mathrm{H}$ & $\mathrm{K}$ & $\mathrm{H}$ & $\mathrm{L}$ & $\mathrm{P}$ & $\mathrm{K}$ \\
\hline $\mathrm{L}$ & $\mathrm{H}$ & $\mathrm{L}$ & I & $\mathrm{H}$ & $\mathrm{L}$ & $\mathrm{H}$ & $\mathrm{K}$ \\
\hline $\mathrm{L}$ & $\mathrm{H}$ & $\mathrm{P}$ & I & $\mathrm{H}$ & $\mathrm{P}$ & $\mathrm{L}$ & $\mathrm{K}$ \\
\hline $\mathrm{L}$ & $\mathrm{H}$ & $\mathrm{H}$ & $\mathrm{K}$ & $\mathrm{H}$ & $\mathrm{P}$ & $\mathrm{P}$ & $\mathrm{R}$ \\
\hline $\mathrm{P}$ & $\mathrm{L}$ & $\mathrm{L}$ & I & $\mathrm{H}$ & $\mathrm{P}$ & $\mathrm{H}$ & $\mathrm{R}$ \\
\hline $\mathrm{P}$ & $\mathrm{L}$ & $\mathrm{P}$ & I & $\mathrm{H}$ & $\mathrm{H}$ & $\mathrm{L}$ & $\mathrm{K}$ \\
\hline $\mathrm{P}$ & $\mathrm{L}$ & $\mathrm{H}$ & I & $\mathrm{H}$ & $\mathrm{H}$ & $\mathrm{P}$ & $\mathrm{R}$ \\
\hline $\mathrm{P}$ & $\mathrm{P}$ & $\mathrm{L}$ & $\mathrm{K}$ & $\mathrm{H}$ & $\mathrm{H}$ & $\mathrm{H}$ & $\mathrm{R}$ \\
\hline $\mathrm{P}$ & $\mathrm{P}$ & $P$ & $\mathrm{~K}$ & & & & \\
\hline
\end{tabular}

TABLE II. INFERENCE RULES OF THE FN-DSON

\begin{tabular}{|c|c||c||}
\hline $\boldsymbol{L}_{\boldsymbol{d}}(\boldsymbol{s}, \boldsymbol{n})$ & $\boldsymbol{L}_{d}(\mathbf{s}, \boldsymbol{n})$ & $\boldsymbol{D}_{d}(\boldsymbol{s}, \boldsymbol{n})$ \\
\hline \hline $\mathrm{L}$ & $\mathrm{L}$ & $\mathrm{I}$ \\
\hline $\mathrm{L}$ & $\mathrm{P}$ & $\mathrm{I}$ \\
\hline $\mathrm{L}$ & $\mathrm{H}$ & $\mathrm{I}$ \\
\hline $\mathrm{P}$ & $\mathrm{L}$ & $\mathrm{I}$ \\
\hline $\mathrm{P}$ & $\mathrm{P}$ & $\mathrm{K}$ \\
\hline $\mathrm{P}$ & $\mathrm{H}$ & $\mathrm{K}$ \\
\hline $\mathrm{H}$ & $\mathrm{L}$ & $\mathrm{I}$ \\
\hline $\mathrm{H}$ & $\mathrm{P}$ & $\mathrm{R}$ \\
\hline $\mathrm{H}$ & $\mathrm{H}$ & $\mathrm{R}$ \\
\hline
\end{tabular}

The proposed Fuzzy Neural approach is compared against a reference scheme in which the AC admits the traffic of a tenant $n$ in a cell $s$ until reaching the planned level $T_{p}(s, n)$, while all the excess traffic exceeding this value is rejected.

To illustrate the behavior of the proposed approach, let consider first a situation in which the offered traffic of $\mathrm{T} 1$ is $24.6 \mathrm{Mb} / \mathrm{s}$ in both cells (i.e., it is above the planned level for this tenant). This value corresponds to the average offered traffic considered by the FN-cSON, while the FN-dSON operates based on average traffic measurements done at a shorter time scale. Specifically, each FN-cSON averaging period corresponds to $10 \mathrm{FN}-\mathrm{dSON}$ averaging periods. Fig. 4 presents the aggregated throughput obtained by each tenant in each cell when varying the offered traffic of $\mathrm{T} 2$, assumed equally distributed in the two cells. It is observed that, when the traffic of T2 is below its planned level of $18.5 \mathrm{Mb} / \mathrm{s}, \mathrm{T} 1$ benefits from a high throughput thanks to the fact that the FN controllers allow that this tenant consumes the resources left unused by T2. Instead, with the reference scheme the throughput of $\mathrm{T} 1$ is limited by the planned value (i.e. 12.5 $\mathrm{Mb} / \mathrm{s}$ ) regardless of the offered traffic of $\mathrm{T} 2$. Throughput improvements of up to $95 \%$ are observed for T1 with respect to the reference scheme. In turn, when the offered traffic of T2 increases and approaches the planned level, it can be observed that the proposed FN controllers allow that each tenant gets a

\footnotetext{
${ }^{1}$ The setting of $m_{R}$ for tenant $s$ in cell $n$ is based on the assumption that, for an offered traffic $T(s, n)>T_{p}(s, n)$, the reference blocking probability to reject the excess traffic above $T_{p}(s, n)$ should be $1-T_{p}(s, n) / T(s, n)$. Then the $m_{R}(s, n)$ is set by bounding this value to a lower limit of 0.04 in order to be above $m_{K}$.
}

throughput in accordance with its expected planned level. Therefore, the results in Fig. 4 reflect that the proposed FN approach is able to efficiently share the available capacity among tenants, ensuring for high loads a capacity share based on the planned levels derived from the SLAs and ensuring for low loads that available resources are efficiently utilized.

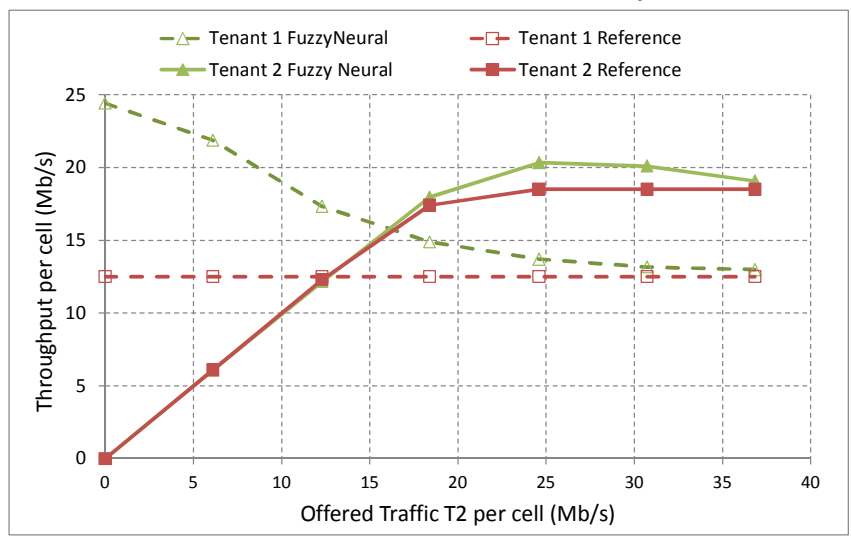

Fig. 4. Total throughput per tenant as a function of the total offered traffic of tenant $\mathrm{T} 2$.

To gain further insight into the operation of the proposed approach, let consider now different situations of the offered traffic of each tenant and cell, given by the traffic mixes of Table III. Traffic mix A corresponds to the case where the offered traffic of both tenants equals the planned level in both cells. In turn, traffic mixes $\mathrm{B}$ and $\mathrm{C}$ represent the situation in which T1's traffic is above the planned level while T2's traffic is below the planned level in both cells. Finally, mixes D and E represent situations in which the offered traffic of each tenant is heterogeneously distributed in the different cells.

TABLE III. OFFERED TRAFFIC (MB/s) FOR DIFFERENT TRAFFIC MIXES

\begin{tabular}{|c|c|c|c|c|}
\hline \multirow{2}{*}{ Traffic Mix } & \multicolumn{2}{|c|}{ Cell 1 } & \multicolumn{2}{c|}{ Cell 2 } \\
\cline { 2 - 5 } & T1 & T2 & T1 & T2 \\
\hline A & 12.5 & 18.5 & 12.5 & 18.5 \\
\hline B & 25 & 6 & 25 & 6 \\
\hline C & 25 & 12 & 25 & 12 \\
\hline D & 19 & 12 & 6 & 25 \\
\hline E & 37 & 6 & 6 & 25 \\
\hline
\end{tabular}

Fig. 5 presents the aggregated throughput of the two tenants across all the cells for the considered traffic mixes. In addition to the comparison with the reference scheme, results also include the comparison between the hybrid SON approach that uses both the FN-cSON and the FN-dSON as described in previous sections and a purely centralized approach that only makes use of the FN-cSON. In this case, the outcome of the FN-cSON controller is the value of blocking probability enforced by the AC, i.e. $P_{B}(s, n)=P_{B, \max }(s, n)$.

Results of Fig. 5 reflect that, for the traffic mix A, in which the offered load of all tenants is in accordance with the planned level, there are very small differences (i.e. less than 4\%) between the considered approaches. On the contrary, for the other traffic mixes, which reflect different types of heterogeneities between tenants in one cell (i.e. mixes B and C) or between different cells (i.e. mixes $\mathrm{D}$ and $\mathrm{E}$ ), there are substantial gains (up to 56\%) in the throughput obtained by the hybrid SON with respect to the reference scheme.

It can also be observed in Fig. 5 that the hybrid SON approach also improves the performance in comparison with 
the purely centralized SON approach, with throughput enhancements of up to $14 \%$. This gain is achieved thanks to the additional flexibility provided by the FN-dSON to adapt the blocking probability below the limit set by the FN-cSON depending on the existing local conditions in each cell.

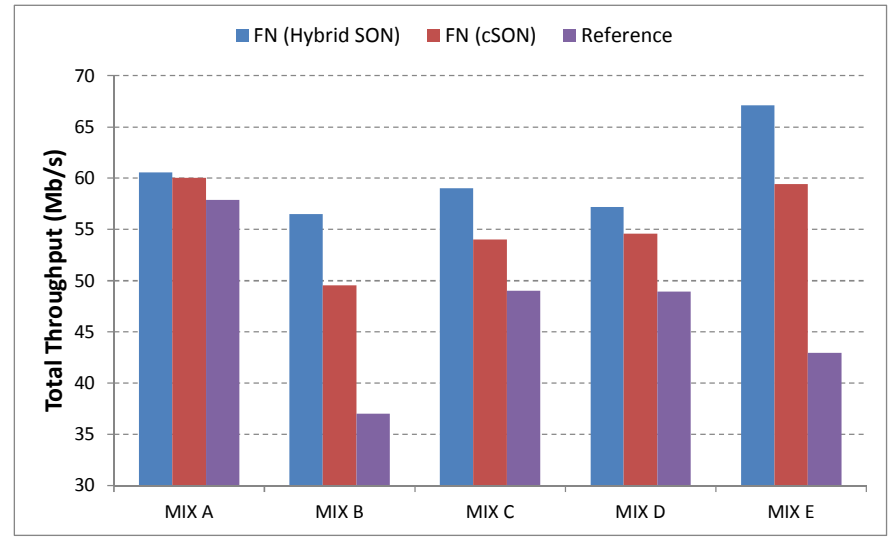

Fig. 5. Total aggregated throughput for different traffic mixes.

\section{CONCLUSIONS}

This paper has proposed a novel strategy for capacity sharing in multi-tenant wireless networks that regulates the amount of traffic of each tenant that can be admitted in each cell. The proposed approach relies on a hybrid SON solution composed by the combination of a centralized FN-cSON running at the management systems and a decentralized FNdSON running at each cell. The FN-cSON operates in the long term and establishes a limit in the maximum blocking probability to be applied to each tenant in each cell based on the overall traffic distribution across the whole scenario. In turn, the FN-dSON operates in a shorter term time scale and selects the actual probability to be applied within the limits established by the FN-cSON. Finally, an AC algorithm enforces the selected blocking probability.

The paper has presented a detailed description of the different components involved in the proposed approach and has provided some results to gain insight on the obtained performance. Results show that the proposed approach is able to smartly adjust the admitted traffic of each tenant depending on the existing traffic distribution in each cell and correspondingly to achieve higher throughput values. Throughput improvements of up to $95 \%$ have been observed with respect to a baseline reference that admits the traffic in each cell based on the planned levels specified for each tenant. In addition, results have also revealed that the joint operation of the cSON and the dSON components of the hybrid approach lead to gains (i.e. up to $14 \%$ ) with respect to a pure centralized approach.

\section{ACKNOWLEDGEMENT}

This work has been supported by the EU funded H2020 5G-PPP project SESAME under the grant agreement 671596 and by the Spanish Research Council and FEDER funds under RAMSES grant (ref. TEC2013-41698-R).

\section{REFERENCES}

[1] P. Rost, et al. "Mobile Network Architecture Evolution toward 5G", IEEE Communication Magazine, May, 2016.
[2] K. Samdanis, X. Costa-Perez, V. Sciancalepore, "From Network Sharing to Multi-Tenancy: The 5G Network Slice Broker", IEEE Communications Magazine, pp. 32-39, July, 2016.

[3] R. Kokku, R. Mahindra, H. Zhang, S. Rangarajan, "NVS: A substrate for Virtualizing Wireless Resources in Cellular Networks", IEEE/ACM Transactions on Networking, Vol. 20, No. 5, October, 2012.

[4] T. Guo, R. Arnott, "Active LTE RAN Sharing with Partial Resource Reservation", IEEE VTC Fall, 2013.

[5] R. Mahindra, M. Khojastepour, H. Zhang, S. Rangarajan, "Radio Access Networks Sharing in Cellular Networks", 21st IEEE International Conference on Network Protocols (ICNP), 2013

[6] P. Caballero Garces, X. Costa-Perez, K. Samdanis, A. Banchs, "RMSC: A Cell Slicing Controller for Virtualized Multi-tenant Mobile Networks", IEEE VTC Spring, 2015.

[7] C.F. Kwong, T. C. Chuah, S.W. Tan, "The ANFIS handover trigger scheme: The Long Term Evolution (LTE) perspective", IEEE Int. Conf. on Fuzzy Systems, 2014.

[8] M.Singh, A. Prakash, D.K. Anvekar, M. Kapoor, R. Shorey, "Fuzzy Logic Based Handoff in Wireless Networks", Proceedings of the VTC 2000 Spring conference, pp. 2375-2379.

[9] N.D.Tripathi, J.H.Reed, H.F.VanLandingham, "Adaptive Handoff Algorithms for Cellular Overlay Systems Using Fuzzy Logic", IEEE 49th Vehicular Technology Conference, VTC 1999

[10] S. V. Truong, L.L. Hung, H. N. Thanh, "A Fuzzy Logic Call Admission Control Scheme inmulti-class traffic Cellular Mobile Networks", Int. Symp. on Computer, Communication, Control and Automation, 2010.

[11] S.Shen, C.J.Chang, C.Y.Huang, Q.Bi, "Intelligent Call Admission Control for Wideband CDMA Cellular Systems", IEEE Transactions on Wireless Communications, Vol.3, No.5, Sept 2004

[12] P. R. Chang and B. C. Wang, "Adaptive fuzzy power control for CDMA mobile radio systems", IEEE Transactions on Vehicular Technology, vol. 15, pp.225-236, May 1996

[13] L. Giupponi, R. Agustí, J. Pérez-Romero, O. Sallent, "A Novel Approach for Joint Radio Resource Management Based on Fuzzy Neural Methodology", IEEE Transactions on Vehicular Technology, Vol. 57, No. 3, May, 2008.

[14] B. Sas, et al. "Self-Optimisation of admission control and handover parameters in LTE”, IEEE VTC Spring, 2011.

[15] H. Klessig, G. Fettweis, "Adaptive Admission Control in InterferenceCoupled Wireless Data Networks: A Planning and Optimization Tool", IEEE ICC 2014.

[16] M. Boujelben, S. Ben Rejeb, S. Tabbane, "A Novel Self-Organizing Scheme for 4G Advanced Networks and Beyond", Int. Symposium on Networks, Computers and Communications, 2014.

[17] F. Lei, L. Wenjing, Q. Xuesong, “A Novel Self-Organized Optimization for Wireless Network Nodes CAC Mechanism" IFIP/IEEE International Symposium on Integrated Network Management, 2013.

[18] K. T. Dinh, S. Kuklinski "Joint Implementation of Several LTE-SON Functions", Globecom workshop on Management of Emerging Networks and Services, 2013.

[19] 3GPP 32.500 v13.0.0, "Self-Organizing Networks (SON); Concepts and requirements (Release 12)", January, 2016.

[20] Small Cell Forum, "SON API for small cells", Document 083.05.01, March, 2015.

[21] C-T. Lin, C.S.G. Lee, "Neural-Network-Based Fuzzy Logic Control and Decision System", IEEE Trans. on Computers, Vol. 40, No. 12, Dec. 1991.

[22] T.J. Ross., Fuzzy Logic with Engineering Applications, McGraw-Hill, 1995.

[23] 3GPP TR 36.814 v9.0.0, "E-UTRA; Further advancements for E-UTRA physical layer aspects (Release 9)", March, 2010.

[24] 3GPP TR 36.942 v12.0.0, "Radio Frequency (RF) system scenarios", September, 2014. 